\title{
Brain Morphology of Cannabis Users With or Without Psychosis: A Pilot MRI Study
}

\author{
Giuseppe Delvecchio ${ }^{1}$, Lucio Oldani $^{2}$, Gian Mario Mandolini ${ }^{2}$, Alessandro Pigoni ${ }^{3}$, Valentina Ciappolino ${ }^{2}$, Giandomenico \\ Schiena $^{2}$, Matteo Lazzaretti ${ }^{2}$, Elisabetta Caletti ${ }^{2}$, Viviana Barbieri ${ }^{1}$, Claudia Cinnante ${ }^{4}$, Fabio Triulzi ${ }^{1,4}$, Paolo Brambilla ${ }^{1,2}$ \\ ${ }^{1}$ Department of Pathophysiology and Transplantation, University of Milan ${ }^{2}$ Department of Neurosciences and Mental Health, Fondazione IRCCS Ca' \\ Granda Ospedale Maggiore Policlinico ${ }^{3}$ MoMiLab Research Unit, IMT School for Advanced Studies Lucca ${ }^{4}$ Neuroradiology Unit, Fondazione IRCCS Ca' \\ Granda Ospedale Maggiore Policlinico \\ *These authors contributed equally
}

\section{Corresponding Author}

Paolo Brambilla

paolo.brambilla1@unimi.it

\section{Citation}

Delvecchio, G., Oldani, L., Mandolini, G.M., Pigoni, A., Ciappolino, V., Schiena, G., Lazzaretti, M., Caletti, E., Barbieri, V., Cinnante, C., Triulzi, F., Brambilla, P. Brain Morphology of Cannabis Users With or Without Psychosis: A Pilot MRI Study. J. Vis. Exp. (162), e60881, doi:10.3791/60881 (2020).

DOI

$10.3791 / 60881$
URL

jove.com/video/60881

\section{Abstract}

Cannabis is the illicit drug most commonly used worldwide, and its consumption can both induce psychiatric symptoms in otherwise healthy subjects and unmask a florid psychotic picture in patients with a prior psychotic risk. Previous studies suggest that chronic and long-term cannabis exposure may exert significant negative effects in brain areas enriched with cannabinoid receptors. However, whether brain alterations determined by cannabis dependency will lead to a clinically significant phenotype or to a psychotic outbreak at some point of an abuser's life remains unclear. The aim of this study was to investigate morphological brain differences between chronic cannabis users with cannabis-induced psychosis (CIP) and non-psychotic cannabis users (NPCU) without any psychiatric conditions and correlate brain deficits with selective socio-demographic, clinical and psychosocial variables.

3T magnetic resonance imaging (MRI) scans of 10 CIP patients and 12 NPCU were acquired. The type of drug, the frequency, and the duration, as well socio-demographic, clinical and psychosocial parameters of dependency were measured. CIP patients had extensive grey matter (GM) decreases in right superior frontal gyrus, right precentral, right superior temporal gyrus, insula bilaterally, right precuneus, right medial occipital gyrus, right fusiform gyrus, and left hippocampus in comparison to chronic cannabis users without psychosis. Finally, in CIP patients, the results showed a negative correlation between a domain of the Brief Psychiatric Rating Scale (BPRS), BPRS-Activity, and selective GM volumes. Overall, the results suggest that cannabis-induced psychosis is characterized by selective brain reductions 
that are not present in NPCU. Therefore, neuroimaging studies may provide a potential ground for identifying putative biomarkers associated with the risk of developing psychosis in cannabis users.

\section{Introduction}

According to the European Monitoring Center for Drugs and Drug Addiction, around 96 million (or 29\%) of adults (aged 15-64) in the European Union are estimated to have tried illicit drugs, especially cannabis, during their life. When considering the youngest and most vulnerable part of the general population, an estimated $16 \%$ of young adults (aged 15-34) used cannabis in the last year, with a male to female ratio of about $2: 1^{1}$. Importantly, cannabis use seems to lead to the development of psychiatric symptoms in healthy subjects, such as mood alterations, increased anxiety, racing thoughts, distorted perceptions, difficulty in thinking and problem solving, ongoing problems with learning and memory, slow reaction time, and loss of control ${ }^{2}$. Such signs and symptoms, though, are normally transient and do not outline a psychiatric condition per se or the need of a treatment. However, cannabis, through its principal psychoactive constituent, named tetrahydrocannabinol (THC), can also induce positive psychotic symptoms including suspiciousness, paranoid delusions, disorders of thought processes, and perceptual alterations $^{3}$, as well as negative symptoms similar to those observed in schizophrenia, such as blunted affect, apathy, avolition, lack of spontaneity, lack of interest, passivity, and cognitive deficits (e.g., memory, executive function, abstract ability, decision making, and attention $)^{3}$. Therefore, at the present time, there is evidence that cannabis consumption can both induce transient psychiatric symptoms in otherwise healthy subjects and unmask a florid psychotic picture in patients with a prior psychotic risk $^{3}$. However, whether this relationship is causal, or purely correlational, is still controversial and debated ${ }^{4}$. Indeed, despite epidemiological studies suggesting a relationship between heavy cannabis consumption and risk of psychosis ${ }^{5}$, the worldwide increased incidence of cannabis use is not accompanied by an augmented incidence of psychosis ${ }^{4}$. This paradox could be explained by the presence of specific confounding differences between cannabis abusers, with early onset of use, daily assumption of high-potency cannabis, and consumption of synthetic cannabinoids carrying the greatest psychotic risk $^{3}$. Moreover, some genetic factors, such as the presence of specific catechol-O-methyltransferase (COMT) polymorphisms, may also confer an augmented vulnerability to develop psychotic symptoms after cannabis exposure in a small proportion of users ${ }^{6}$.

In this regard, human neuroimaging studies attempted to investigate the potential neural mechanisms through which cannabis may lead to psychotic symptoms ${ }^{7}$, since preclinical studies previously showed that THC is active within brain areas rich in cannabinoid type 1 receptors (CB1R), including hippocampus, amygdala, striatum, and prefrontal cortex $(\mathrm{PFC})^{8}$. Indeed, experimental THC administration to healthy cannabis users have been shown to attenuate ventrostriatal activation during a learning task and concurrently induce psychotic symptoms $^{9}$ as well as altered prefrontal-striatal activation during attentional salience processing ${ }^{10}$. With regard to structural magnetic resonance imaging (MRI) studies, some authors detected significant grey matter (GM) volume reductions in the prefrontal cortex ${ }^{11,12,13}$, the hippocampus ${ }^{14,15}$, the amygdala ${ }^{16}$ and the putamen ${ }^{17}$ in regular cannabis users compared to nonusers while others did not report any significant brain differences between 
these two groups $18,19,20,21$ or reported increased GM volumes within the medial temporal, the amygdala, the hippocampus, the posterior cingulate and the cerebellum among adolescents with low cannabis use ${ }^{22}$.

Furthermore, few studies explored whether there are any specific brain differences between cannabis users with psychotic symptoms and cannabis users without any psychiatric conditions. One functional MRI study compared healthy subjects who did and did not experience psychotic symptoms after THC consumption and it reported increased activity during a go/no-go task in the right middle temporal gyrus and decreased activity in both parahippocampal and fusiform gyri, which was also associated with greater inhibition errors only in the psychotic group $^{23}$. In contrast, Epstein and Kumra found that both psychotic and nonpsychotic adolescents with cannabis use disorder shared similar brain alterations; specifically, they detected attenuated cortical thinning in the left superior frontal gyrus, the right pars triangularis, the left pars opercularis, the left and right supramarginal gyri, the left and right inferior parietal cortices and the left superior temporal gyrus in both groups ${ }^{24}$. In a previous study, the same authors compared adolescents with early onset schizophrenia (EOS) with (EOS+) and without (EOS-) cannabis use disorder (CUD), adolescents with CUD only and healthy controls ${ }^{25}$ . Interestingly, they detected smaller grey matter volumes in the left superior parietal region in both EOS- and CUD groups compared to healthy controls. However, they did not find additive volumetric alterations in adolescents with EOS+ compared to other groups. Finally, a more recent and larger study found a significant total effect from lifetime cannabis consumption to psychotic-life experiences in a sample of adolescents. Interestingly, the authors found an association between psychotic-life experiences and reduced expansion within the uncus of the right hippocampus/ parahippocampus $^{26}$.

Therefore, these studies, although not all concordant, suggest that cannabis-induced psychosis may be characterized by neurobiological deficits, similar to those detected in pure psychotic disorders. However, whether brain alterations determined by cannabis dependency and highlighted by neuroimaging investigations will lead to a clinically significant phenotype or to a psychotic outbreak at some point of an abuser's life still remains unclear. In this regard, the investigation of brain morphology among psychotic cannabis users in comparison with cannabis users without any psychiatric symptoms could be of paramount importance in order to understand the neurobiological underpinnings of cannabis-induced psychosis. However, to the best of our knowledge, so far no studies have compared cannabis-induced psychotic subjects with healthy cannabis users in terms of brain structural morphology and clinical parameters, such as psychopathology, frequency and duration of dependency, quality of life, personality traits, childbirth complication and childhood abuse. In this context, the aim of this study is to investigate morphological brain differences between chronic cannabis users with substanceinduced psychosis (CIP) and non-psychotic cannabis users (NPCU) and to correlate brain deficits with selective socio-demographic, clinical and psychosocial variables. We hypothesized that CIP patients will show significant reductions in GM volumes compared to NPCU as well as possible correlations between GM volumes and sociodemographic, clinical and psychosocial scales.

\section{Protocol}

10 CIP patients and 12 NPCU were recruited for this study. All patients were recruited at the psychiatric inward of the 
University Policlinico Hospital of Milan, Italy, whereas the cannabis users were enrolled in the Milan catchment area. All patients were in stable pharmacological treatment. Either leftor right- handed participants were included. All participants had a habitual cannabis consumption and the type of drug, the frequency and the duration, as well socio-demographic, clinical and psychosocial parameters of dependency were measured. The study was approved by the local ethical committee.

\section{Participants}

1. Use the following inclusion criteria: For patients: age 18-45 years old, DSM-IV diagnosis of Cannabis-induced Psychotic Disorder, heavy cannabis consumption at the time of the study and in the previous 6 months. For NPCU: age 18-45 years old, no DSM-IV diagnosis, heavy cannabis consumption at the time of the study and in the previous 6 months.

2. Use the following exclusion criteria: a diagnosis of mental retardation, any current major medical or neurological illness, a history of traumatic head injury with loss of consciousness, and any other Axis I, including alcohol abuse, or Axis II disorders and pregnancy. Verify that psychotic symptoms do not precede the onset of the cannabis use and do not persist for a substantial period of time after the cessation of acute withdrawal or severe intoxication. Verify that there is no history of recurrent nonsubstance-related episodes.

3. To obtain informed consent read the consent form to the participants. Have both the participant and the investigator sign the consent form in duplicate. Store the consent form for records.
4. To evaluate the diagnosis of CIP patients, use the Structured Clinical Interview for Diagnosis (SCID-I) of the Diagnostic and Statistical Manual of Mental Disorders, 4th edition, text revision (DSM-IV-TR) ${ }^{27}$.

5. To establish the frequency and the duration of dependency, use the manual for the semistructured clinical interview for children and adolescents SCICA ${ }^{28}$.

\section{Clinical and psychosocial evaluation}

NOTE: Several clinical and psychosocial scales were administered to all the participants.

1. To evaluate psychiatric symptoms, use the Brief Psychiatric Rating Scale (BPRS) ${ }^{29}$, the Young Mania Rating Scale $(\mathrm{YMRS})^{30}$, the Montgomery-Åsberg Depression Rating Scale (MADRS) ${ }^{31}$, the Hamilton Depression Rating Scale (HAM-D) ${ }^{32}$ and the Hamilton Anxiety Rating Scale (HAM-A) ${ }^{33}$.

2. To explore the presence of trauma or infection during or immediately after the partum, use the Murray-Lewis Obstetric Complications Scale (MLOCS) ${ }^{34}$.

3. To assess experiences of neglect or abuse, use the Childhood Experience of Care and Abuse Questionnaire $(\mathrm{CECA}-\mathrm{Q})^{35}$.

4. To estimate the Socio-economic status (SES), use the Socio Economic Status Scale of MacArthur ${ }^{36}$

5. Use the Neighbourhood Scale (NS) ${ }^{37}$ to assess the specific characteristics of the neighbourhood, in terms of neighbourhood satisfaction (NS-A), sense of security (NS-B), level of degradation (NS-C), willingness on the part of fellow citizens to intervene in adverse situations (NS-D), and degree of acceptance of substances (NS-E). 
6. Employ the Temperament and Character Inventory (TCl-125) for exploring personality traits ${ }^{38,39}$.

7. To assess the quality of life and the global functioning use the Manchester Short Assessment of Quality of Life $(\text { MANSA })^{40}$ and the Quality of Life Index (QL-index $)^{41}$ and the Global Assessment of Functioning (GAF) ${ }^{27}$ scales, respectively.

NOTE: All socio-demographic and clinical data are summarized in Table 1.

\section{Magnetic resonance imaging}

1. Insert the participant in a supine position on the bed of the 3 Tesla MRI scanner.

2. Place a radio frequency (RF) coil over the participant's head.

3. Provide earplugs and headphones to block background noise.

4. Attach foam pads to immobilize the head.

5. Instruct the subject to remain still.

6. Run MRI session from the workstation in the control room.

1. Run a 3-plane gradient echo scan for alignment and localization and perform a shim procedure to generate a homogeneous, constant magnetic field.

2. Start an echo-planar-imaging protocol for MRI. The acquisition parameters for the acquisition of highresolution T1-weighted three-dimensional brain scan are already set in the imaging program and should not be changed. The parameters are: repetition time [TR] $=9.8$, echo time $[\mathrm{TE}]=4.6 \mathrm{~ms}$, in plane voxel size $=$ $0.9375 \times 0.9375$, matrix $=256 \times 256$, flip angle $=8^{\circ}$.
7. Remove the participant from the MR scanner room. Transfer the MR data to disk and close the session. NOTE: A total of 185 contiguous $1 \mathrm{~mm}$ sagittal slices extending superiorly from the inferior aspect of the cerebellum to encompass most of the brain were selected from a sagittal localizer scan.

\section{Pre-processing steps}

NOTE: A voxel-based morphometry analysis should be performed using Statistical Parametric Mapping (SPM12) implemented in MATLAB.

1. Perform the following pre-processing steps, shown in the Script_pre-processing script file, before carrying out group analyses.

1. Segmentation: Process the structural image to distinguish and separate the white matter tissues, the grey matter tissues and the cerebrospinal fluid into different images. This separation is obtained thanks to the combination of probability maps, elaborated from the general knowledge of tissue distribution combined with model cluster analyses that identifies voxel distributions of specific tissues in the original image. Run the segment.mat batch file.

2. DARTEL (Diffeomorphic Anatomical Registration Through Exponentiated Lie algebra) tools: determine the nonlinear deformations for registering the GM and white matter images of all participants. Run the create_template.mat batch file.

3. Normalization: during the spatial normalization phase, adapt MRI images to an anatomical standard template. This is because every subject has little differences in the form and organization of the brain such as the size and morphologic differences in 
structures. Run the normalize_to_MNI.mat batch file.

4. Spatial Smoothing: after motion correction, perform an isotropic Gaussian kernel of $6 \mathrm{~mm}$ full width at half maximum Gaussian kernel to increase the signalto-noise ratio and to account for subtle variations in anatomic structures. Run the normalize_to_MNI.mat batch file.

5. Extract the total intracranial volume (ICV) using SPM12: it can be obtained by adding up the density values in GM, white matter, and CSF class images and multiplying by the voxel volumes.

NOTE: Once the pre-processing is completed, it is possible to elaborate the data.

NOTE: Please refer to the SPM manual (https:// www.fil.ion.ucl.ac.uk/spm/doc/spm12_manual.pdf) that provides a detailed description of the preprocessing steps employed in this study and the SPM commands to use. Please also refer to the script and Matlab batches included in the supplementary materials with the exact pre-processing steps used for this study.

\section{Statistical analyses}

1. Perform chi-square tests (categorical variables) and two sample $t$ tests (quantitative variables) for exploring differences between the two groups on demographic, clinical and psychosocial scale.

2. Perform a one-way Analysis of Variance (ANOVA), in the context of a General Linear Model (GLM) design to compare GM volumes between CIP patients and NPCU. Gender and age were used as controlling variables in all the analyses. Run the one-way ANOVA batch file.
3. Carry out whole-brain regression analyses, only for the CIP group, to explore whether the scores in all the clinical and psychosocial scales employed in this study were significantly correlated with GM volumes changes. Do not use any brain mask but consider all voxels. Run the Regression analysis batch file with the clinical scale of interest.

4. Convert stereotactic coordinates of the peak maxima of the suprathreshold clusters from the MNI spatial array (www.mni.mcgill.ca) to that of Talairach and Tournoux ${ }^{42}$. NOTE: In all the neuroanatomical analyses, the volumetric differences among subjects were considered by proportional scaling for the total intracranial volume (ICV).

1. For the ANOVA, set the significance threshold to $p$ $<0.001$ uncorrected, with a minimum cluster size of $k=30$, whereas for the multiple regression analyses, a $p<0.05$ peak Family-Wise Error ( $p F W E$ ) corrected was considered significant and a minimum cluster size of $k=10$ was employed. The former threshold was considered due to the small sample size employed in this study and therefore the results emerged from this analysis must be considered as preliminary. The latter threshold is more stringent since the $p$-value is corrected for multiple comparisons.

NOTE: Please refer to the VBM8 Manual for more details about postprocessing steps (http://dbm.neuro.uni-jena.de/ vbm8/VBM8-Manual.pdf). Please also refer to the Matlab batches named "one-way ANOVA" and "Regression analysis" included in the supplementary materials with the exact model used for this study. Due to the exploratory nature of this study, a formal 
sample size calculation would have been of little value and therefore it was not performed.

\section{Representative Results}

\section{Socio-demographic, clinical and psychosocial results}

There were no differences in terms of gender $(x 2=0.6$, $p=0.4)$, age $(t=-0.21 ; p=0.83)$, age of onset of dependency $(t=-0.79 ; p=0.44)$ and educational level $(t=1.21 ; p=0.24)$ between CIP patients and NPCU. However, some differences between the two groups were observed in one temperament dimension (Harm Avoidance, $\mathrm{t}=3.71 ; \mathrm{p}=0.001$ ) and onecharacter dimension (Self-Transcendence, $t=2.94 ; p=0.008$ ) of the $\mathrm{TCl}$ where CIP patients showed higher scores compared to NPCU. Finally, NPCU also showed higher scores compared to CIP patients in one sub-dimension of the Neighborhood Scale (NS-E) $(t=-3.55 ; p=0.002)$, in the SES total $(t=-2.13 ; p=0.046)$, in the Quality of Life-Index $(t=-8.1 ; p=0.0001)$, in the GAF $(t=-4.71 ; p=0.0001)$ and in one character dimension of the TCl (Self Directedness, $t=-3.97$; $\mathrm{p}=0.001)$.

Specifically, for CIP, the frequency of cannabis dependency was daily for 9 subjects (90\%) and several times a week for 1 subject (10\%). Instead, the frequency of cannabis dependency in the NPCU group was daily for 7 subjects $(60 \%)$, several times a week for 4 subjects $(30 \%)$, and multiple times a month for 1 subject (10\%). The mean age of onset of dependency was at 18 years old for CIP patients and at 16 years old for the NPCU group. Although all participants were taking cannabis, some CIP patients $(\mathrm{N}=6)$ and NPCU $(\mathrm{N}=3)$ also reported previous use of other drugs, including cocaine, LSD and heroin/methadone, but with lower frequency than cannabis. The frequency of cannabis use did not differ between the two groups $\left(x^{2}=1.69, p=0.42\right)$. Moreover, no statistical difference in type and frequency of cocaine, heroin/ methadone and LSD use was observed between the two groups (cocaine: $x^{2}=0.06, p=0.79$ and $x^{2}=4.1, p=0.39$;

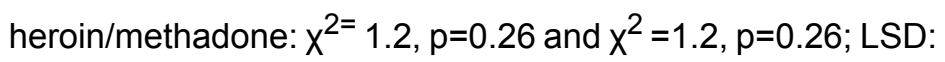
$x^{2}=0.01, p=0.89$ and $x^{2}=2.0, p=0.36$ ). Although we are aware that the presence of poly-consumption in the sample might have negatively affected the generalizability of the findings, it is important to highlight that the use of other drugs was very limited compared to cannabis use. Indeed, in contrast to cannabis use, the consumption of other drugs was lifetime and not occurring during the time of the study. Nonetheless, our results should be taken cautiously and need to be replicated in a more homogeneous sample.

\section{VBM results}

VBM analysis showed that CIP patients had extensive GM decreases compared to NPCU in right superior frontal gyrus ((Brodmann area $[B A]$ 10), right precentral (BA 4), right superior temporal gyrus (BA 22), insula bilaterally (BA13), right precuneus (BA7), right medial occipital gyrus (BA 19), right fusiform gyrus (BA 37) and left hippocampus ( $p<0.001$ uncorrected; Table 2 and Figure 1). No GM differences were observed in NPCU compared to CIP patients.

\section{Correlations between GM regions and clinical scales}

In CIP patients, the results showed a negative correlation between a domain of the BPRS, BPRS-Activity, and selective GM volumes within left superior temporal cortex (BA 38, $x=-40 y=17 z=-35, z=5.9$, cluster size=19) and left cerebellum $(x=-12 y=-36 z=-20, z=6.1$, cluster size=18). Moreover, the same scale was positively correlated with cuneus bilaterally (BA 18; left: $x=-9 y=-90 z=9, z=7.0$, cluster size=24; right: $x=15 y=-85 z=24, z=7.3$, cluster size=13), left inferior occipital gyrus (BA 17; $x=-9 y=-88 z=-6, z=7.4$, cluster size=34), right inferior parietal lobule (BA 40; $x=58 y=-35 z=22, z=6.7$, cluster size=33), right superior prefrontal cortex (BA 9; $x=3 y=51$ 
$z=29, z=6.2$, cluster size $=23$ ) (all $p<0.05$ pFWE corrected). No significant correlations in any of the other clinical scales were observed in CIP patients.

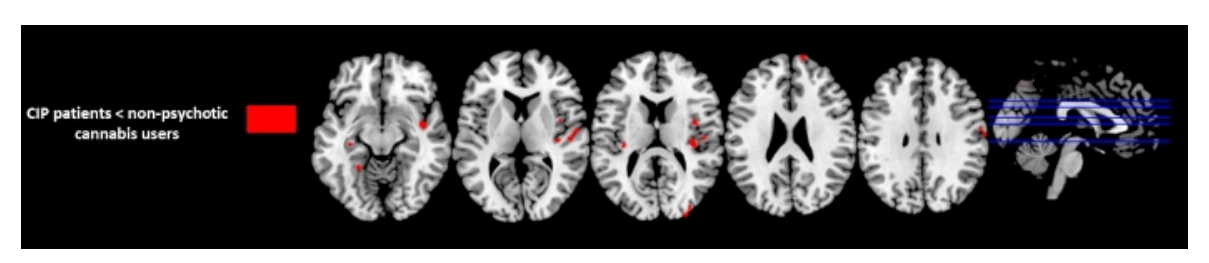

Figure 1: Regions with significant GM difference between Substance-induced psychosis (CIP) patients and nonpsychotic cannabis users $(\mathbf{p}<\mathbf{0 . 0 0 1}$, uncorrected, $\mathbf{k}=\mathbf{3 0}$ ). Please click here to view a larger version of this figure. 


\begin{tabular}{|c|c|c|c|c|}
\hline & CIP patients & $\begin{array}{l}\text { Non-psychotic } \\
\text { cannabis users }\end{array}$ & Statistics & p-value \\
\hline & $n=10$ & $n=12$ & & \\
\hline Age, mean (SD) & $27(9.21)$ & $26(0.89)$ & $t=-0.213$ & $p=0.833$ \\
\hline Sex, male/female & $8 / 2$ & $11 / 1$ & $x 2=0.630$ & $p=0.427$ \\
\hline $\begin{array}{l}\text { Age of onset } \\
\text { of cannabis } \\
\text { use, mean (SD) }\end{array}$ & $18(9.69)$ & $16(1.83)$ & $\mathrm{t}=-0.786$ & $p=0.441$ \\
\hline \multirow[t]{7}{*}{$\begin{array}{l}\text { Type }(\mathrm{N}) \text {; frequency } \\
\text { of other drug use }\end{array}$} & $\begin{array}{l}\text { Cannabis }(N=10) \\
\text { Daily }(N=9) \text {, multiple } \\
\text { times a week }(N=1) \text {. }\end{array}$ & $\begin{array}{l}\text { Cannabis }(\mathrm{N}=12) \text {; Daily } \\
(\mathrm{N}=7) \text {, multiple times } \\
\text { a week }(\mathrm{N}=4) \text {, multiple } \\
\text { times a month }(\mathrm{N}=1) \text {. }\end{array}$ & Frequency: $x^{2}=1.69$, & $p=0.42$ \\
\hline & \multirow{2}{*}{$\begin{array}{c}\text { Cocaine }(\mathrm{N}=4) \\
\text { multiple times a } \\
\text { week }(\mathrm{N}=2) \text {, multiple } \\
\text { times a month }(\mathrm{N}=2) \text {. }\end{array}$} & \multirow{2}{*}{$\begin{array}{l}\text { Cocaine }(N=3) \text {; multiple } \\
\text { times a week }(N=1) \text {, } \\
\text { multiple times a month } \\
\qquad(N=1) \text {, less than } \\
\text { one a month }(N=1) \text {. }\end{array}$} & Type: $x^{2}=0.06$ & Type: $p=0.79$ \\
\hline & & & Frequency: $x^{2}=4.1$ & Frequency: $p=0.39$ \\
\hline & \multirow{2}{*}{$\begin{array}{l}\text { Heroin/Methadone } \\
\qquad(\mathrm{N}=1) \text {; multiple } \\
\text { times a week. }\end{array}$} & \multirow{2}{*}{$\begin{array}{l}\text { No Heroin/ } \\
\text { Methadone users. }\end{array}$} & Type: $x^{2}=1.2$ & Type: $p=0.26$ \\
\hline & & & Frequency: $x^{2}=1.2$ & Frequency: $p=0.26$ \\
\hline & \multirow{2}{*}{$\begin{array}{l}\text { LSD }(\mathrm{N}=1) \text {; less } \\
\text { than one a month. }\end{array}$} & \multirow{2}{*}{$\begin{array}{l}\text { LSD }(N=1) \text {; multiple } \\
\text { times a month. }\end{array}$} & Type: $x^{2}=0.01$ & Type: $p=0.89$ \\
\hline & & & Frequency: $x^{2}=2.0$ & Frequency: $p=0.36$ \\
\hline $\begin{array}{l}\text { Age of onset, } \\
\text { mean (SD) }\end{array}$ & $25(8.46)$ & - & - & - \\
\hline BPRS TOT, mean (SD) & $43(9)$ & $20(3)$ & $t=8.860$ & $p=0.0001$ \\
\hline Anxiety-Depression & $10(5)$ & $6(2)$ & $t=2.629$ & $p=0.016$ \\
\hline Anergia & $8(3)$ & $4(1)$ & $\mathrm{t}=3.284$ & $p=0.004$ \\
\hline Thought Disorders & $12(3)$ & $4(0)$ & $t=9.754$ & $p=0.0001$ \\
\hline Activity & $6(2)$ & $3(0)$ & $t=4.557$ & $p=0.0001$ \\
\hline
\end{tabular}




\begin{tabular}{|c|c|c|c|c|}
\hline $\begin{array}{c}\text { Hostility- } \\
\text { Suspiciousness }\end{array}$ & $8(4)$ & $3(0)$ & $t=4.053$ & $p=0.001$ \\
\hline HAM-D, mean (SD) & $11(6.42)$ & $4(4.96)$ & $t=3.258$ & $p=0.004$ \\
\hline HAM-A, mean (SD) & $11(6.62)$ & $3(3.93)$ & $t=3.487$ & $p=0.002$ \\
\hline MADRS, mean (SD) & $14(7.76)$ & $6(6.35)$ & $t=2.635$ & $p=0.016$ \\
\hline YMRS, mean (SD) & $13(7.92)$ & $0(1.44)$ & $t=5.378$ & $p=0.0001$ \\
\hline \multicolumn{5}{|l|}{ CECA-Q, mean (SD) } \\
\hline CECA-QMA & $13(5.20)$ & $13(3.89)$ & $t=-0.069$ & $p=0.946$ \\
\hline CECA-QMN & $19(5.83)$ & $19(4.64)$ & $t=-0.284$ & $p=0.779$ \\
\hline CECA-QPA & $14(6.44)$ & $14(5.56)$ & $t=-0.130$ & $p=0.990$ \\
\hline CECA-QPN & $24(11.69)$ & $24(7.12)$ & $\mathrm{t}=0.070$ & $p=0.945$ \\
\hline \multicolumn{5}{|l|}{$\begin{array}{c}\text { Neighbourhood } \\
\text { scale }^{*} \text {, mean (SD) }\end{array}$} \\
\hline NS- A & $9(1.78)$ & $8(2.23)$ & $t=0.782$ & $p=0.443$ \\
\hline NS- B & $6(2.50)$ & $7(1.56)$ & $t=-1.070$ & $p=0.298$ \\
\hline NS- C & $9(5.87)$ & $10(7.66)$ & $t=-0.265$ & $p=0.794$ \\
\hline NS-D & $6(2.31)$ & $5(1.53)$ & $t=1.378$ & $p=0.183$ \\
\hline NS-E & $3(1.35)$ & $4(0.29)$ & $t=-3.546$ & $p=0.002$ \\
\hline SES ${ }^{* *}$ total, mean (SD) & $33.6(12.60)$ & $45.3(13.05)$ & $t=-2.132$ & $p=0.046$ \\
\hline Study & $11.3(4.22)$ & $15.3(5.93)$ & $t=-1.800$ & $p=0.087$ \\
\hline Occupation & $22.3(10.39)$ & $30.0(8.79)$ & $t=-1.885$ & $p=0.074$ \\
\hline QL - Index, mean (SD) & $6(1.65)$ & $10(0.62)$ & $t=-8.098$ & $p=0.0001$ \\
\hline GAF, mean (SD) & $58(15.21)$ & $83(9.68)$ & $t=-4.715$ & $p=0.0001$ \\
\hline MANSA, mean (SD) & $54(14.16)$ & $61(6.01)$ & $t=-1.250$ & $p=0.226$ \\
\hline \multicolumn{5}{|l|}{ TCI, mean (SD) } \\
\hline $\mathrm{TCl} \mathrm{Ns}$ & $59.92(10.75)$ & $55.95(12.86)$ & $t=0.173$ & $p=0.864$ \\
\hline $\mathrm{TCl} \mathrm{Ha}$ & $55.67(7.71)$ & $45.61(5.68)$ & $t=3.708$ & $p=0.001$ \\
\hline $\mathrm{TCl} \mathrm{Rd}$ & $48.67(10.41)$ & $50.49(9.02)$ & $t=-0.668$ & $p=0.512$ \\
\hline
\end{tabular}




\begin{tabular}{|c|c|c|c|c|}
\hline TCI P & $49.82(11.49)$ & $39.32(8.83)$ & $\mathrm{t}=2.033$ & $\mathrm{p}=0.056$ \\
\hline TCI Sd & $28.64(11.85)$ & $49.89(7.42)$ & $\mathrm{t}=-3.969$ & $\mathrm{p}=0.001$ \\
\hline TCI Co & $42.15(12.21)$ & $49.07(5.60)$ & $\mathrm{t}=-1.430$ & $\mathrm{p}=0.008$ \\
\hline TCI St & $65.56(12.34)$ & $50.82(8.16)$ & $\mathrm{t}=2.940$ & \\
\hline
\end{tabular}

Table 1: Socio-demographic, clinical and psychosocial variables of the whole sample. BPRS (Brief Psychiatric Rating Scale); CECA-Q (Childhood Experience of Care and Abuse Questionnaire); CIP (Cannabis-Induced Psychosis); GAF (Global Assessment of Functioning); HAM-A (Hamilton Anxiety Rating Scale); MADRS (Montgomery-Asberg Depression Rating Scale); HAM-D (Hamilton Depression Rating Scale); MANSA (Manchester Short Assessment of Quality of Life); NSA (Neighbourhood satisfaction); NS-B (Feelings of safety); NS-C (Neighbourhood incivilities); NS-D (Collective efficacy); NS-E (Cannabis acceptance); SD (Standard Deviation); SES (Socio Economic Status); QL-Index (Quality of Life-Index); ); TCI (Temperament and Character Inventory); TCI Ns (Novelty Seeking); TCI Ha (Harm Avoidance); TCI Rd (Reward Dependence); TCI P (Persistence); TCI Sd (Self Directedness); TCI Co (Cooperativeness); TCI St (Self Transcendence); YMRS (Young Mania Rating Scale). * NS-A ranges from 0 to 16, where 16 represented extreme satisfaction with the area of residence; NS-B ranges from 0 to 8 , where 8 represented a strong feeling of safety; NS-C ranges from 0 to 32 , where 32 indicated a high level of incivilities; NS-D ranges from 0 to 12, where 8 represented a high level of collective efficacy amongst neighbours; NS-E ranges from 'agree strongly' (score of 4) to 'disagree strongly' (score of 0 ). ${ }^{* *}$ Lower levels of schooling are associated to lower scores while higher levels of schooling are associated to higher scores (ie. Less than $7^{\text {th }}$ grade $=$ 3; Graduate degree $=21$ ). Similarly, Occupations with lower cognitive engagement are associated to lower scores, while occupations requiring more cognitive resources are associated to higher scores (Farm worker= 5; Physician= 45). 


\begin{tabular}{|c|c|c|c|c|c|c|c|c|}
\hline \multirow[t]{2}{*}{ Gyrus } & \multirow[t]{2}{*}{ BA } & \multirow[t]{2}{*}{ Laterality } & \multicolumn{3}{|c|}{ MNI coordinates } & \multirow[t]{2}{*}{ Cluster size } & \multirow[t]{2}{*}{ z-values } & \multirow{2}{*}{$\begin{array}{l}\text { Cohen's } d \\
\text { effect size }\end{array}$} \\
\hline & & & \multicolumn{3}{|c|}{$x y z$} & & & \\
\hline \multicolumn{8}{|c|}{ CIP patients < non-psychotic cannabis users } & \\
\hline $\begin{array}{l}\text { Superior } \\
\text { Frontal }\end{array}$ & 10 & Right & 13 & 65 & 22 & 38 & 3.4 & $-1,26$ \\
\hline Precentral & 4 & Right & 59 & -5 & 26 & 61 & 3.8 & $-0,83$ \\
\hline $\begin{array}{l}\text { Superior } \\
\text { Temporal }\end{array}$ & 22 & Right & 62 & -7 & 3 & 146 & 4.2 & $-0,60$ \\
\hline Insula & 13 & Right & 36 & -21 & 13 & 142 & 4.1 & $-0,43$ \\
\hline Insula & 13 & Left & -33 & -23 & 14 & 32 & 3.8 & $-0,46$ \\
\hline Precuneus & 7 & Right & 6 & -66 & 50 & 41 & 3.7 & $-0,51$ \\
\hline $\begin{array}{c}\text { Medial } \\
\text { Occipital }\end{array}$ & 19 & Right & 33 & -86 & 21 & 80 & 4 & $-0,84$ \\
\hline Fusiform & 37 & Left & -25 & -47 & -8 & 32 & 3.7 & $-0,29$ \\
\hline Hippocampus & - & Left & -33 & -22 & -5 & 36 & 3.8 & $-0,68$ \\
\hline \multicolumn{8}{|c|}{ Non-psychotic cannabis users $<$ CIP patients } & \\
\hline & & & upr & clus & & & & \\
\hline
\end{tabular}

Table 2: VBM results. Brain regions showing significant reduced grey matter volumes between the CIP patients and nonpsychotic cannabis users $(\mathrm{P}<0.001$ uncorrected). BA (Brodmann area); CIP (Cannabis-Induced Psychosis); MNI (Montreal Neurological Institute)

\section{Discussion}

In the present study, we observed that only the presence of psychotic symptoms discriminated the detection of brain morphological alterations. Indeed, chronic cannabis users with CIP showed decreased GM volumes mainly in the prefronto-temporo-limbic network compared to nonpsychotic cannabis users (NPCU). Moreover, regarding the psychometric questionnaires, correlations between the domain BPRS-Activity and selective GM volumes have been highlighted. Specifically, we observed a negative correlation between such BPRS scale and left superior temporal cortex and left cerebellum together with a positive correlation with the cuneus bilaterally, the left inferior occipital gyrus, the right inferior parietal lobule and the right superior prefrontal cortex. However, we should mention that the lack of a control group of healthy subjects with no cannabis dependency prevented us from exploring if cannabis use caused brain alterations or not. 
In general, the results are not surprising since previous MRI studies showed that psychotic disorders, such as schizophrenia, shared similar GM abnormalities, especially in frontal and temporo-limbic regions ${ }^{29}, 30$. However, it is still unclear why some chronic cannabis users developed psychotic symptoms while others remained healthy. Indeed, in the sample, we only detected small clinical differences between the two groups and therefore the extensive GM abnormalities observed in the CIP group may not be associated with their specific clinical profile. Specifically, 9 of 10 CIP patients reported a daily cannabis use compared to 7 of 12 in the NPCU group. Moreover, no differences in terms of age, gender, age of onset of cannabis use and educational level were found between the two groups. However, we should consider that this lack of differences could be due to the small sample size that also limited the possibility to statistically analyse and interpret these factors. One hypothesis is that the psychotic process itself is responsible for the decrease in brain volume, regardless cannabis use. Indeed, previous studies showed no GM differences between psychotic patients with and without cannabis consumption, thus finding no clear evidence for cannabis use to be related to GM alterations in first episode psychotic patients ${ }^{45}$. However, cannabis use may have contributed to brain alterations and subsequently induced psychosis only in a subgroup of susceptible cannabis users.

The first hypothesis is in line with those studies showing brain abnormalities in psychotic disorders. Specifically, the results showed that CIP patients compared to NPCU had extensive GM volume decreases in some brain areas known to be involved in emotional regulation, such as frontotemporal cortices, insula, hippocampus, and fusiform gyrus $^{46}$. Interestingly, disruptions in these structures, especially in prefrontal regions, might explain the mood instability and greater emotional reactivity in adolescents and young adults, as well as impulsive behaviors and substance-seeking 33,34 . Indeed, it has been consistently reported that emotion regulation/processing are associated with recruitment of a set of prefrontal brain regions involved in cognitive control over emotional limbic structures. For example, greater difficulties in emotional regulation among tobacco smokers have been associated to a weaker connectivity between inferior frontal gyrus and amygdala compared to non-smokers ${ }^{49}$. Therefore, it might be plausible that among CIP patients the development of psychotic symptoms was associated with interfered balance between these structures.

Additionally, we observed that the group of CIP patients showed disruptions in the dorsolateral prefrontal cortex (DLPFC), a key region involved in major cognitive functions, including working memory, executive functions ${ }^{50}$ and emotional regulation ${ }^{51}$. Indeed, this finding is not surprising since the DLPFC works together with risk-monitoring regions, such as the insula (a key structure of the salience network recently found to be involved in addiction ${ }^{52}$ ), which has been also found altered in the group of CIP patients, and anterior cingulate cortex, ultimately suggesting that impairments in distinguishing risky from safe choices may result from a disruption between DLPFC and such riskmonitoring regions ${ }^{53}$.

Moreover, CIP patients showed a GM volume decrease in the superior temporal cortex. Interestingly, this result is in line with the evidence reported by a previous multimodal neuroimaging study ${ }^{54}$, which employed a larger sample of CIP patients $(\mathrm{N}=16)$, the majority of whom overlap with the sample employed in this study that found extensive GM alteration in temporal cortices in CIP patients. Overall 
such evidence further confirms the key role of the superior temporal cortex in psychosis, since the involvement of this structure has been consistently reported in abilities often found disrupted in psychotic patients, including language processing and theory of mind abilities ${ }^{39,40}$. Also, the results aligned with previous evidence reporting the association between volume reductions of this region and auditory hallucination or thought disorders 41,42 as well as with a previous MRI study suggesting the disruption of this area in substance dependent individuals compared to healthy controls $^{59}$.

Finally, a significant GM volume decrease in hippocampus in CIP patients emerged from the results. Such finding is in line with previous evidence showing structural and functional changes in this structure in early psychoses and in atrisk mental state/first-episode psychosis, in comparison to healthy controls $60,61,62,63$. Normal hippocampal function is required for a number of mental functions including memory and emotional behaviour 48,49 and it has been proposed that reduced volume in this structure may represent a marker of a negative clinical outcome in patients with a firstepisode psychosis ${ }^{66}$. However, in contrast to the results, hippocampal deficits have been also reported in young and adult cannabis users, who have been found to have thinner cortices and reduced volumes in this region $67,68,69,70$. Therefore, a clear picture on the role of the hippocampus in substance abuse has still not be attained. Nonetheless, the results point towards the hypothesis that the cortico-limbic system is compromised in the group of CIP patients, as also suggested by a previous MRI study ${ }^{54}$ and might explain the emotional elaboration deficits, which has been proposed to be a critical precursor of future psychotic development ${ }^{55,56}$, often observed in these patients.
Therefore, it seems reasonable to hypothesize that cannabisinduced psychosis is associated with brain alterations in regions within the prefronto-temporo-limbic network, which may therefore represent a common neurodevelopmental substrate of multiple forms of psychosis. Interestingly, longitudinal studies proposed that some brain disruptions, including smaller orbitofrontal cortex volumes ${ }^{73}$, increased fronto-parietal and decreased visual association regions activation, as well as cognitive deficits, such as poorer executive functions ${ }^{74}$, may be present even before the initiation of cannabis dependency. Therefore, it might be that these individuals with underlying brain alterations are more likely to develop psychotic symptoms after cannabis use initiation. In addition, there is evidence reporting that possessing risk alleles in AKT1 and DRD2 genes, which are involved in dopamine signalling, is associated with increased risk of developing psychosis after cannabis use ${ }^{3}$. Therefore, the detection of morphological decreases in CIP patients could reflect an augmented genetic susceptibility to the neurotoxic effect of chronic cannabis use in this group of subjects.

Finally, in the CIP group, the results also showed a negative correlation between a sub-domain of the BPRS, the BPRS-Activity, and selective GM volumes within left superior temporal cortex and left cerebellum. Also, this subscale was positively correlated with cuneus bilaterally, left inferior occipital gyrus, right inferior parietal lobule, and right superior prefrontal cortex. In general, although correlations between clinical symptomatology and GM structures have been extensively reported, especially in schizophrenia ${ }^{75}$, the results are still heterogeneous, with a mixed picture of inverse ${ }^{76}$, positive ${ }^{77}$ or no ${ }^{78}$ correlations between selective GM volumes and clinical scales. Notably, the negative correlation observed between BPRS-Activity and 
superior temporal cortex seems to be in line with previous MRI evidence showing inverse correlations between this structure and positive symptom severity ${ }^{76}$, ultimately further suggesting the key role of this structure in the production of psychotic symptoms. Similarly, the positive correlation found between BPRS-Activity and superior prefrontal cortex seems to agree with other MRI studies reporting a similar correlation between negative symptoms and GM volumes with the prefrontal cortex ${ }^{79}$.

As a whole, the results of the current study provide preliminary evidence on the presence of significant association between brain alteration and severity of psychopathology.

The current study suffers from some limitations. First, all psychotic patients were taking pharmacological treatments that could have influenced the results. Second, the lack of a control group formed by healthy subjects not exposed to cannabis does not allow a further comparison with the two groups of cannabis users (psychotic and not). In addition, although the two groups were very similar in terms of number of subjects (10 CIP patients vs $12 \mathrm{NPCU})$, the small sample size limits the significance of the results achieved and therefore must be considered as preliminary. Further limitations are strictly connected to the nature of the population investigated. Indeed, some patients with CIP (6/10) and a rather small proportion of NPCU (3/12) had a lifetime history of other substance consumption (i.e., cocaine, LSD and heroin/methadone). Moreover, we did not examine the genetic alleles linked to addiction, which could have helped to discriminate the two groups. Nonetheless, the cannabis consumption, although assessed in terms of frequency and volume and duration with a specific tool ${ }^{20}$, was not uniform across the two groups. Finally, in this study we did not explore brain activation and we did not assess the neurocognitive state of the sample. Therefore, the lack of these information could have affected the results since previous studies demonstrated the presence of selective brain dysfunctions in patients with schizophrenia with substance abuse in the medial prefrontal cortex, the orbitofrontal cortex and the amygdala as well as a better premorbid neurocognitive profile with a greater long-term decline compared to the same patients without substance abuse $^{80}$. Therefore, further functional MRI studies exploring brain activity coupled with neuropsychological assessments on larger samples and with homogenous consumption habits are needed to confirm our results.

According to our results, cannabis-induced psychosis may be characterized by GM volume decreases in selective brain structures. Therefore, in view of the crucial and comprehensive role of the endocannabinoid system in the brain, the increasing prevalence of cannabis use, its chronic use during neurodevelopment, as well as the progressively higher $\mathrm{THC}$ concentration in the current market, it seems mandatory to clarify which aspects of cannabis exposure (e.g., age at initiation, quantity, frequency, and duration) determine the greatest risk for the progression towards psychotic-related disorders. However, whether reductions in prefronto-temporo-limbic regions represent a substrate of the psychotic process itself or a direct consequence of cannabis exposure among susceptible subjects remains a complex issue. In this context, the methods employed in the study could be useful to better characterize the neurobiological and clinical features of cannabis-induced psychosis. Finally, longitudinal neuroimaging studies taking into account also potential confounding factors, such as cannabis dose, potency, THC/Cannabidiol ratio, frequency of use, age of onset, familiar history of psychosis, and genetic polymorphisms may provide a potential ground for identifying 
putative biomarkers which may ultimately help clinicians to detect those cannabis users that are more likely to develop psychosis.

\section{Disclosures}

None.

\section{Acknowledgments}

None.

\section{References}

1. European Monitoring Centre for Drugs and Drug Addiction European Drug Report 2019. European Drug Report 2019: Trends and Developments. Publications Office of the European Union, Luxembourg. (2019).

2. De Aquino, J.P. et al. The Psychiatric Consequences of Cannabinoids. Clinical Therapeutics. 40 (9), 1448-1456 (2018).

3. Murray, R.M. et al. Cannabis-associated psychosis: Neural substrate and clinical impact. Neuropharmacology. 124, 89-104 (2017).

4. Gage, S.H. Cannabis and psychosis: triangulating the evidence. The Lancet Psychiatry. 6 (5), 364-365 (2019).

5. Gage, S.H., Hickman, M., Zammit, S. Association between cannabis and psychosis: Epidemiologic evidence. Biological Psychiatry. 79 (7), 549-556 (2016).

6. Burns, J.K. Pathways from Cannabis to Psychosis: A Review of the Evidence. Frontiers in Psychiatry. 4, 128 (2013).

7. Bhattacharyya, S., Atakan, Z., Martin-Santos, R., Crippa, J.A., McGuire, P.K. Neural mechanisms for the cannabinoid modulation of cognition and affect in man: a critical review of neuroimaging studies. Current Pharmaceutical Design. 18 (32), 5045-54 (2012).

8. Atkinson, D.L., Abbott, J.K. Cannabinoids and the Brain: The Effects of Endogenous and Exogenous Cannabinoids on Brain Systems and Function. The Complex Connection Between Cannabis and Schizophrenia. Academic Press. (2018).

9. Bhattacharyya, S. et al. Modulation of Mediotemporal and Ventrostriatal Function in Humans by $\Delta 9$ Tetrahydrocannabinol. Archives of General Psychiatry. 66 (4), 442-451 (2009).

10. Bhattacharyya, S. et al. Induction of psychosis by $\Delta 9$-tetrahydrocannabinol reflects modulation of prefrontal and striatal function during attentional salience processing. Archives of General Psychiatry. 69 (1), 27-36 (2012)

11. Battistella, G. et al. Long-term effects of cannabis on brain structure. Neuropsychopharmacology. 39 (9), 2041-2048 (2014).

12. Price, J.S., McQueeny, T., Shollenbarger, S., Browning, E.L., Wieser, J., Lisdahl, K.M. Effects of marijuana use on prefrontal and parietal volumes and cognition in emerging adults. Psychopharmacology. 232 (16), 2939-2950 (2015)

13. Filbey, F.M. et al. Long-term effects of marijuana use on the brain. Proceedings of the National Academy of Sciences of the United States of America. 111 (47), 16913-16918 (2014).

14. Yücel, M. et al. Regional brain abnormalities associated with long-term heavy cannabis use. Archives of General Psychiatry. 65 (6), 694-701 (2008). 
15. Lorenzetti, V. et al. Gross morphological brain changes with chronic, heavy cannabis use. British Journal of Psychiatry. 206 (1), 77-78 (2015).

16. Schacht, J.P., Hutchison, K.E., Filbey, F.M. Associations between cannabinoid receptor-1 (CNR1) variation and hippocampus and amygdala volumes in heavy cannabis users. Neuropsychopharmacology. 37 (11), 2368-2376 (2012).

17. Yip, S.W. et al. Pretreatment measures of brain structure and reward-processing brain function in cannabis dependence: An exploratory study of relationships with abstinence during behavioral treatment. Drug and Alcohol Dependence. 140, 33-41 (2014).

18. Medina, K.L. et al. Prefrontal cortex morphometry in abstinent adolescent marijuana users: Subtle gender effects. Addiction Biology. 14 (4), 457-468 (2009).

19. DeLisi, L.E. et al. A preliminary DTI study showing no brain structural change associated with adolescent cannabis use. Harm Reduction Journal. 3 (1), 17 (2006).

20. Jager, G. et al. Effects of frequent cannabis use on hippocampal activity during an associative memory task. European Neuropsychopharmacology. 17 (4), 289-297 (2007).

21. Tzilos, G.K. et al. Lack of hippocampal volume change in long-term heavy cannabis users. American Journal on Addictions. 14 (1), 64-72 (2005).

22. Orr, C. et al. Grey matter volume differences associated with extremely low levels of cannabis use in adolescence. Journal of Neuroscience. 39 (10), 1817-1827 (2019).

23. Atakan, Z. et al. Cannabis affects people differently: Inter-subject variation in the psychotogenic effects of $\Delta 9$ tetrahydrocannabinol: A functional magnetic resonance imaging study with healthy volunteers. Psychological Medicine. 43 (6), 1255-1267 (2013).

24. Epstein, K.A., Kumra, S. Altered cortical maturation in adolescent cannabis users with and without schizophrenia. Schizophrenia Research. 162 (1-3), 143-152 (2015).

25. Kumra, S. et al. Parietal lobe volume deficits in adolescents with schizophrenia and adolescents with cannabis use disorders. Journal of the American Academy of Child and Adolescent Psychiatry. 51 (2), 171-180 (2012).

26. Yu, T. et al. Cannabis-Associated Psychotic-like Experiences Are Mediated by Developmental Changes in the Parahippocampal Gyrus. Journal of the American Academy of Child and Adolescent Psychiatry. 59 (5), 642-649 (2019).

27. American Psychiatric Association. Diagnostic and statistical manual of mental disorders (4th ed., text rev.). Washington. (2000).

28. McConaughy, S.H., Achenbach, T.M. Manual for the semistructured clinical interview for children and adolescents. ASEBA (2001).

29. Overall, J.E., Gorham, D.R. The Brief Psychiatric Rating Scale. Psychological Reports. 10 (3), 799-812 (1962).

30. Young, R.C., Biggs, J.T., Ziegler, V.E., Meyer, D.A. A rating scale for mania: Reliability, validity and sensitivity. British Journal of Psychiatry. 133 (5), 429-435 (1978).

31. Montgomery, S.A., Asberg, M. A new depression scale designed to be sensitive to change. British Journal of Psychiatry. 134 (4), 382-389 (1979). 
32. Hamilton, M. A rating scale for depression. Journal of Neurology, Neurosurgery, and Psychiatry. 23 (1), 56 (1960).

33. Hamilton, M. Hamilton Anxiety Rating Scale (HAM-A). Journal of Medicine. 61 (4), 81-82 (1959).

34. Lewis, S.W., Murray, R.M. Obstetric complications, neurodevelopmental deviance, and risk of schizophrenia. Journal of Psychiatric Research. 21 (4), 413-421 (1987).

35. Bifulco, A., Bernazzani, O., Moran, P.M., Jacobs, C. The childhood experience of care and abuse questionnaire (CECA.Q): Validation in a community series. British Journal of Clinical Psychology. 44 (4), 563-581 (2005).

36. Adler, N.E., Stewart, J. The MacArthur Scale of Subjective Social Status. MacArthur Research Network on SES and Health. (2007).

37. Hur, M., Nasar, J.L., Chun, B. Neighborhood satisfaction, physical and perceived naturalness and openness. Journal of Environmental Psychology. 30 (1), 52-59 (2010).

38. Cloninger, C.R., Svrakic, D.M., Przybeck, T.R. A Psychobiological Model of Temperament and Character. Archives of General Psychiatry. 50 (12), 975-990 (1993).

39. Delvecchio, G. et al. Normative data and effects of age and gender on temperament and character dimensions across the lifespan in an Italian population: A crosssectional validation study. Journal of Affective Disorders. 204, 83-91 (2016).

40. Priebe, S., Huxley, P., Knight, S., Evans, S. Application and results of the Manchester Short Assessment of Quality of Life (MANSA). International Journal of Social Psychiatry. 45 (1), 7-12 (1999).
41. Spitzer, W.O. et al. Measuring the quality of life of cancer patients. A concise QL-Index for use by physicians. Journal of Chronic Diseases. 34 (12), 585-597 (1981).

42. Talairach, J. Tournoux, P. Co-planar stereotaxic atlas of the human brain: 3-dimensional proportional system : an approach to cerebral imaging. Thieme, NY. (1988).

43. Ellison-Wright, I., Glahn, D.C., Laird, A.R., Thelen, S.M., Bullmore, E. The anatomy of first-episode and chronic schizophrenia: An anatomical likelihood estimation metaanalysis. American Journal of Psychiatry. 165 (8), 1015-1023 (2008).

44. Fornito, A., Yücel, M., Patti, J., Wood, S.J., Pantelis, C. Mapping grey matter reductions in schizophrenia: An anatomical likelihood estimation analysis of voxelbased morphometry studies. Schizophrenia Research. 108 (1-3), 104-113 (2009).

45. Haller, S. et al. Combined grey matter VBV and white matter TBSS analysis in young first episode psychosispatients with and without cannabis consumption. Brain Topography. 26 (4), 641-647 (2013).

46. Hou, J. et al. Review on neural correlates of emotion regulation and music: Implications for emotion dysregulation. Frontiers in Psychology. 8, 501 (2017).

47. Martin, R.E., Ochsner, K.N. The Neuroscience of Emotion Regulation Development: Implications for Education. Current Opinion in Behavioral Sciences. 10, 142-148 (2016).

48. Pfeifer, J.H., Allen, N.B. Arrested development? Reconsidering dual-systems models of brain function in adolescence and disorders. Trends in Cognitive Sciences. 16 (6), 322-9 (2012). 
49. Faulkner, P., Dean, A.C., Ghahremani, D.G., London, E.D. Neural Basis of Smoking-Related Difficulties in Emotion Regulation. International Journal of Neuropsychopharmacology. (2020).

50. Dedoncker, J., Brunoni, A.R., Baeken, C., Vanderhasselt, M.A. A Systematic Review and Meta-Analysis of the Effects of Transcranial Direct Current Stimulation (tDCS) Over the Dorsolateral Prefrontal Cortex in Healthy and Neuropsychiatric Samples: Influence of Stimulation Parameters. Brain Stimulation. 9 (4), 501-517 (2016).

51. Sturm, V.E., Haase, C.M., Levenson, R.W. Emotional Dysfunction in Psychopathology and Neuropathology: Neural and Genetic Pathways. Genomics, Circuits, and Pathways in Clinical Neuropsychiatry. 345-364 (2016).

52. Koob, G.F., Volkow, N.D. Neurobiology of addiction: a neurocircuitry analysis. The Lancet Psychiatry. 3 (8),760-773 (2016).

53. Kohno, M., Morales, A.M., Guttman, Z., London, E.D. A neural network that links brain function, white-matter structure and risky behavior. Neurolmage. 149, 15-22 (2017).

54. Altamura, A.C. et al. Structural and metabolic differentiation between bipolar disorder with psychosis and substance-induced psychosis: An integrated MRI/ PET study. European Psychiatry. 41, 85-94 (2017).

55. Anderson, J.E. et al. An MRI study of temporal lobe abnormalities and negative symptoms in chronic schizophrenia. Schizophrenia Research. 58 (2-3), 123-34 (2002).

56. Brüne, M., Brüne-Cohrs, U. Theory of mind--evolution, ontogeny, brain mechanisms and psychopathology.
Neuroscience and Biobehavioral Reviews. 30 (4), 437-55 (2006).

57. Takahashi, T. et al. Morphologic alterations of the parcellated superior temporal gyrus in schizophrenia spectrum. Schizophrenia Research. 83 (2-3), 131-43 (2006).

58. Holinger, D.P. et al. Superior temporal gyrus volume abnormalities and thought disorder in left-handed schizophrenic men. The American Journal of Psychiatry. 156 (11), 1730-5 (1999).

59. Ersche, K.D., Jones, P.S., Williams, G.B., Turton, A.J., Robbins, T.W., Bullmore, E.T. Abnormal brain structure implicated in stimulant drug addiction. Science. 335 (6068), 601-4 (2012).

60. Baglivo, V. et al. Hippocampal Subfield Volumes in Patients With First-Episode Psychosis. Schizophrenia Bulletin. 44 (3), 552-559 (2018).

61. Pruessner, M. et al. Reduced hippocampal volume and hypothalamus-pituitary-adrenal axis function in first episode psychosis: Evidence for sex differences. Neurolmage: Clinical. 7, 195-202 (2015).

62. Walter, A. et al. Hippocampal volume in subjects at high risk of psychosis: A longitudinal MRI study. Schizophrenia Research. 142 (1-3), 217-222 (2012).

63. Verma, S. et al. Hippocampal Volumes in First-Episode Psychosis. The Journal of Neuropsychiatry and Clinical Neurosciences. 21 (1), 24-29 (2009).

64. Anand, K.S., Dhikav, V. Hippocampus in health and disease: An overview. Annals of Indian Academy of Neurology. 15 (4), 239 (2012).

65. Wible, C.G. Hippocampal physiology, structure and function and the neuroscience of schizophrenia: a 
unified account of declarative memory deficits, working memory deficits and schizophrenic symptoms. Behavioral Sciences. 3 (2), 298-315 (2013).

66. Sauras, R. et al. Volumetric and morphological characteristics of the hippocampus are associated with progression to schizophrenia in patients with first-episode psychosis. European Psychiatry. 45, 1-5 (2017).

67. Chye, Y. et al. Alteration to hippocampal volume and shape confined to cannabis dependence: a multi-site study. Addiction Biology. 24 (4), 822-834 (2019).

68. Chye, Y. et al. Cannabis-related hippocampal volumetric abnormalities specific to subregions in dependent users. Psychopharmacology. 234 (14), 2149-2157 (2017).

69. Burggren, A.C. et al. Subregional Hippocampal Thickness Abnormalities in Older Adults with a History of Heavy Cannabis Use. Cannabis and Cannabinoid Research. 3 (1), 242-251 (2018).

70. Schacht, J.P., Hutchison, K.E., Filbey, F.M. Associations between cannabinoid receptor-1 (CNR1) variation and hippocampus and amygdala volumes in heavy cannabis users. Neuropsychopharmacology. 37 (11), 2368-76 (2012).

71. Grace, A.A. Gating of information flow within the limbic system and the pathophysiology of schizophrenia. Brain Research. Brain Research Reviews. 31 (2-3), 330-41 (2000).

72. Aleman, A., Kahn, R.S. Strange feelings: Do amygdala abnormalities dysregulate the emotional brain in schizophrenia? Progress in Neurobiology. 77 (5), 283-298 (2005).

73. Cheetham, A. et al. Orbitofrontal volumes in early adolescence predict initiation of cannabis use: A 4-year longitudinal and prospective study. Biological Psychiatry. 71 (8), 684-692 (2012).

74. Tervo-Clemmens, B. et al. Early Cannabis Use and Neurocognitive Risk: A Prospective Functional Neuroimaging Study. Biological Psychiatry: Cognitive Neuroscience and Neuroimaging. 3 (8), 713-725 (2018).

75. Nestor, P.G. et al. Dissociable contributions of MRI volume reductions of superior temporal and fusiform gyri to symptoms and neuropsychology in schizophrenia. Schizophrenia Research. 91 (1-3), 103-6 (2007).

76. Nesvåg, R., Saetre, P., Lawyer, G., Jönsson, E.G., Agartz, I. The relationship between symptom severity and regional cortical and grey matter volumes in schizophrenia. Progress in Neuro-Psychopharmacology and Biological Psychiatry. 33 (3), 482-490 (2009).

77. Lacerda, A.L.T. et al. Morphology of the orbitofrontal cortex in first-episode schizophrenia: relationship with negative symptomatology. Progress in NeuroPsychopharmacology \& Biological Psychiatry. 31 (2), 510-6 (2007).

78. Volkow, N.D. et al. Effects of Cannabis Use on Human Behavior, Including Cognition, Motivation, and Psychosis: A Review. JAMA Psychiatry. 73 (3), 292-7 (2016).

79. Walton, E. et al. Prefrontal cortical thinning links to negative symptoms in schizophrenia via the ENIGMA consortium. Psychological Medicine. 48 (1), 82-94 (2018).

80. Adan, A. et al. Neurobiological underpinnings and modulating factors in schizophrenia spectrum disorders with a comorbid substance use disorder: A systematic review. Neuroscience and Biobehavioral Reviews. 75, 361-377 (2017). 